\title{
NONLINEAR DISTORTION \& ITS REDUCTION TECHNIQUES FOR COHERENT OPTICAL OFDM SYSTEM: A REVIEW
}

\author{
Sunil Joshi ${ }^{1 *}$, Gaurav Sharma ${ }^{2}$ \\ ${ }^{*}$ M. Tech Scholar Faculty of Engg. \& Tech, ECE, Mewar University, Chittorgarh (India) \\ ${ }^{2}$ Assistant Professor \& Head, Faculty of Engg. \& Tech, ECE Mewar University, Chittorgarh (India) \\ smsc2006@gmail.com
}

*Corresponding Author: -

\begin{abstract}
: -
In this paper the literature review is done for different compensation technique to reduce the nonlinear distortion for optical coherent system. Comparative analysis done so for dispersion compensation fiber, method of Fiber Bragg Grating, digital signal processing (DSP), pre and post symmetric-DCF techniques. The digital signal processing is very practical for all type of nonlinear distortion is minimized while dispersion technique is use to compensate the dispersion loss only and other methods are useful to reduce nonlinear distortion.
\end{abstract}

Key words: Nonlinear Distortion, OFDM, Dispersion, and DSP. 


\section{INTRODUCTION}

The Orthogonal frequency division multiplexing (OFDM) has been widely employed into numerous digital standards for broad-range of applications such as digital audio/video broadcasting and wireline /wireless communication systems. Recently it has been shown that OFDM can be applied in optical long haul transmission systems and had many advantages over conventional single-carrier modulation format. In recent times, a worldwide convergence has occurred for the use of Orthogonal Frequency Division Multiplexing) as a promising technology for high data rates. It is a method of encoding digital data on multiple carrier frequencies. In meticulous, many wireless standards (Wi- Max, IEEE802.11a, LTE, DVB) have adopted the OFDM technology as a mean to boost noticeably outlook wireless communications. OFDM is a particular form of multi-carrier transmission and is suited for frequency selective channels and high data rates. This technique transforms a frequency-selective wide-band channel into a group of non-selective narrowband channels, which makes it robust against large delay spreads by preserving orthogonality in the frequency domain. OFDM has developed into a popular scheme for wideband digital communication, used in applications such as digital television and audio broadcasting, DSL Internet access, wireless networks, power line networks, and 4G mobile communications [1].

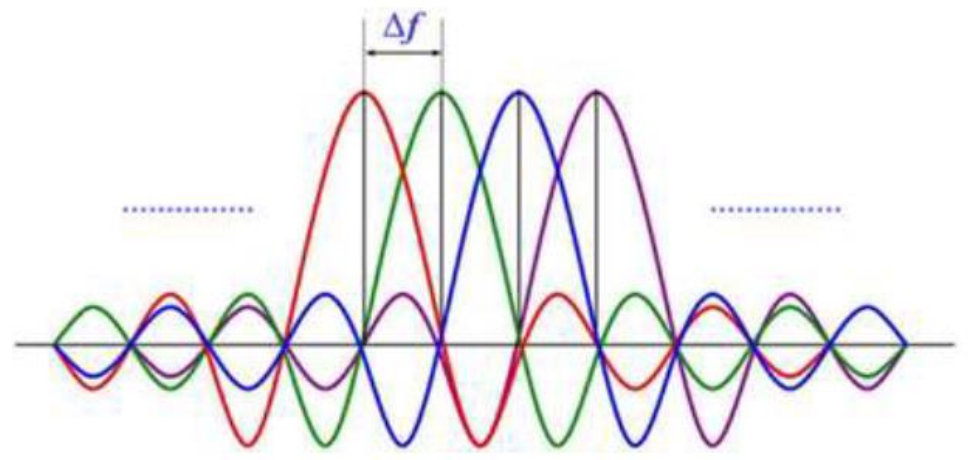

Fig.1 Orthogonal Frequency Division Multiplexing

Many key merits of the OFDM techniques have been studied and proven in the communications industry. Firstly, the frequency spectra of OFDM subcarriers are partially overlapped, resulting in high spectral efficiency. Secondly, the channel dispersion of the transmission system is easily estimated and removed, and thirdly, the signal processing in the OFDM transceiver can take advantage of the efficient algorithm of FFT/IFFT with low computation complexity. Recently, an equivalent optical-domain multi-carrier format, called coherent optical OFDM (CO- OFDM) has been proposed for long haul transmission.

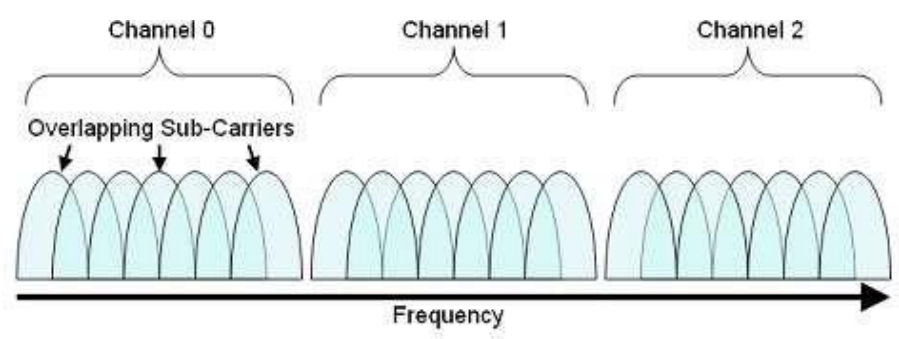

Fig.2 Spectrum of OFDM

Digital signal processing is used at the receiver for compensation of fiber impairments. Digital compensation can be done at the receiver after the optical signal has been converted to electric signal. If the baseband signal is sampled above the Nyquist rate, digitized signal represents the full content of analog electric signal, which enables digital signal processing compensation. DSP has advantages, such as signals can be delayed, split, amplified and manipulated without degradation in signal quality [2].

\section{NONLINEAR DISTORTION OF OPTICAL OFDM}

A Laser is most imperative part of optical system. It is easy to integrate with the other components on a semiconductor substrate, allowing a more compact OFDM system a further cost reduction.

The thermal noise \& shot noise are contributed for total noise for ODFM system. The other sources of noises are channel $\&$ transmitter source those offer the extra noise. The symbiotic combination of Digital Signal Processing, coherent detection, and spectrally efficient modulation formats has resulted in the digital coherent optical receiver. Coherent detection employing multilevel modulation format has become one of the most promising technologies for next generation high-speed transmission system due to the high power and spectral efficiencies. With the powerful DSP, coherent optical receivers allow the significant equalization of chromatic dispersion (CD), polarization mode dispersion (PMD), phase noise (PN) and nonlinear effects in the electrical domain.

Single-mode fibers support the transmission of two polarization-modes that are orthogonal to each other. Thus change in the states-of-polarization is also responsible of distortion because temperature fluctuations and random birefringence due 
to mechanical stress cause the (SOP) and, therefore, the group velocity to vary with time and across the full length of the fiber.The nonlinear impairments are self-phase modulation (SPM) and stimulated Brillouin scattering (SBS)

The symbiotic combination of Digital Signal Processing, coherent detection, and spectrally efficient modulation formats has resulted in the digital coherent optical receiver. Coherent detection employing multilevel modulation format has become one of the most promising technologies for next generation high-speed transmission system due to the high power and spectral efficiencies. With the powerful DSP, coherent optical receivers allow the significant equalization of chromatic dispersion (CD), polarization mode dispersion (PMD), phase noise (PN) and nonlinear effects in the electrical domain [3].

\section{REDUCTION TECHNIQUES}

The different problems or issues of OFDM system like Low SNR, High EVM and overlap constellation diagram. The demand for broadband wireless communications is growing with an extremely rapid pace. These systems are required to be operating in an environment which is characterized by high carrier frequency, data transmission rate and mobility; altogether such an environment can be modelled by a frequency selective fast time varying fading channel. It has been studied and established, that the multicarrier data transmission techniques such as MC-CDMA and OFDM are best suited for such channels.

The OFDM is a special case of multicarrier modulation in which serial stream of data is divided in parallel and then modulated by orthogonal sub-carriers with partial overlapping frequency bands. The OFDM symbols have relatively longtime duration as compared to single carrier modulation with a narrow bandwidth. This increases the robustness against multipath deteriorations and results in less complex equalizers which helps in performing the channel equalization easily in the frequency domain through a bank of one-tap multipliers. The main advantage of using OFDM system is to increase the robustness against frequency selective fading or narrowband interference.

L. Gruner-Nielson proposed the dispersion compensation fiber to overcome the optical impairments. Optical fiber communication systems are limited by fiber loss, chromatic dispersion and nonlinearity [7].

N. Q. Ngo proposed the method of Fiber Bragg Grating, dispersion can be mitigated optically through installing dispersion compensating Fiber (DCF) along the link or employing optical dispersion compensating modules with Fiber Bragg gratings. These solutions add loss to the signal path and are difficult to adapt dynamically to changing network requirements [8].

Y. Jiang and his group give a fundamentally different approach has been developed to mitigate dispersion using digital signal processing (DSP) implemented by an application specific integrated circuit (ASIC) [9]

Very recently the Ali Y. Fattah and their research team presented the leading edge of this DSP revolution in optical communications Digital signal processing in advance optical communication systems is enabled by high-speed digitalto-analog converter (DAC) and analog-to-digital converter (ADC) technology operating at billions of samples per second. Optical Impairments can be effectively mitigated through Digital Signal Processing (DSP) at the transmitter or at the receiver. [10]

Digital signal processing is used at the receiver for compensation of fiber impairments. Digital compensation can be done at the receiver after the optical signal has been converted to electric signal. If the baseband signal is sampled above the Nyquist rate, digitized signal represents the full content of analog electric signal, which enables digital signal processing compensation. DSP has advantages, such as signals can be delayed, split, amplified and manipulated without degradation in signal quality.

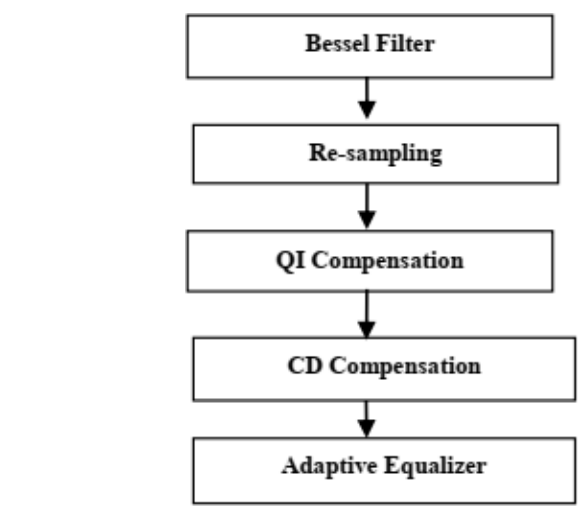

Fig.3 Different Stages of Digital Signal Processing

The electronic signal-processing techniques can be broadly classified as adaptive equalization at the receiver, predistortion at the transmitter, and electric-field domain signal processing. The Electronic Dispersion Compensation (EDC) at the receiver can be most conveniently designed to be fully adaptive and, due to its ease of use and attractive economics, this approach will be emphasized.

Digital signal processing in optical communication systems is enabled by high-speed digital-to-analog converter (DAC) and analog-to-digital converter (ADC) technology operating at billions of samples per second. Fiber chromatic dispersion can be effectively mitigated through electronic dispersion compensation (EDC) at the transmitter or at the receiver 


\section{CONCLUSION}

In this paper we review the different compensation technique to reduce the nonlinear distortion for optical coherent system. Those methods include use of dispersion compensation fiber, method of Fiber Bragg Grating, using digital signal processing (DSP), pre and post symmetric-DCF techniques. The comparative study is done by author contribution that the dispersion technique is use to compensate the dispersion loss only, while the other methods are useful to reduce nonlinear distortion but by using the digital signal processing the all type of nonlinear distortion is minimized.

\section{Acknowledgements}

The authors would like to acknowledgement to P.G. Coordinator, Faculty of Engineering Mewar University for his valuable suggestions \& guides.

\section{REFERENCES}

[1].W. Shieh, H. Bao, and Y. Tang, Coherent optical OFDM: theory and design, Optics Express Vol. 16(2), $2008,842$.

[2].W. Shieh, PMD-Supported Coherent Optical OFDM Systems, IEEE Photonics Technology Letters, Vol. 19, No. 3, 2007, $134-136$.

[3].A. Sangeetha et.al, performance analysis of Dispersion compensation Techniques in a 100 gbps Coherent-optical system, International Journal of Engineering and Technology, Vol 5 (3), 2013, 258-264.

[4].Rajani et.al, "Comparison of Pre-, Post- and Symmetrical-Dispersion Compensation Schemes for 10/15Gbps using Different Modulation Formats at Various Optical Power Levels using Standard and Dispersion Compensated Fibers, International Journal of Computer Applications, Vol. 50(21), 2012.

[5].Ip, E.M. and Kahn, J.M., Fiber Impairment Compensation Using Coherent Detection and Digital Signal Processing, Journal of Lightwave Technology, Vol.4 (2), 2010, $502-519$.

[6].Danish Rafique et.al, "Compensation of intra-channel nonlinear fibre impairments using simplified digital backpropagation algorithm" Optics Express, Vol. 19(10), 2011, 9453-9460.

[7].L. Gruner-Nielson, S. N. Knudsen, B. Edvold, T. Veng, D. Magnussen, C. C. Larsen, and H. Damsgaard, Design and manufacture of dispersion compensating fibre for simultaneous compensation of dispersion and dispersion slope," Proc. Conf. Opt. Fiber Commun., vol. 2,1999, 232-234.

[8].Q. Ngo, S. Y. Li, R. T. Zheng, S. C. Tjin, and P. Shum, electrically tunable dispersion compensator with fixed center wavelength using fiber bragg grating," IEEE J. Lightwave Technology, vol. 21, 2003, 1568-1575.

[9].Y. Jiang, X. Tang, J. C. Cartledge, and K. Roberts, Electronic pre-compensation of narrow optical filtering for OOK, DPSK and DQPSK modulation formats," IEEE J. Lightwave Technol., vol. 27, 2009, 3689-3698.

[10]. Ali Y. Fattah, Zainab Faydh Mohammed, "Electronic Signal Processing for Cancelation of Optical Systems Impairments", IJCCCE, 13(2), 2013, 55-71. 